\title{
Resolution of Inappropriate Sexual Behavior in an Elderly Male with Intellectual Disability with Memantine: Case Report, and Review of the Literature
}

\author{
Zeba Hasan Hafeez ( $\nabla$ zebahafeez@hotmail.com ) \\ Adult and Geriatric Psychiatrist, Kaiser Permanente, Santa Rosa Adjunct Faculty, Touro University, \\ California Touro University Outpatient Psychiatry Director
}

\section{Case report}

Keywords: Inappropriate Sexual Behavior, Intellectual Disability, Memantine, Geriatric Psychiatry

Posted Date: February 12th, 2021

DOl: https://doi.org/10.21203/rs.3.rs-197230/v1

License: (c) (1) This work is licensed under a Creative Commons Attribution 4.0 International License. Read Full License 


\section{Abstract}

\section{Background}

Memantine is a non-competitive $\mathrm{N}$-methyl-d-aspartate receptor antagonist approved for the treatment of moderate to severe Alzheimer's disease (AD). However, given its involvement of glutamate pathways, monotherapy as well as combination with other medications has been found to be effective in a number

of psychiatric conditions ${ }^{(1)}$. Resolution of new onset inappropriate sexual behavior (ISB) with memantine in a 67 year old male with intellectual disability (ID) is presented.

\section{Case presentation}

A 67 year old, white male with ID with a full scale IQ of 68 , was treated in psychiatry long term for intermittent agitation and anxiety. At age 65 , he manifested ISB which was not controlled with non pharmacologic measures and various medications. ISB resolved when memantine was added to his concurrent psychotropics and gradually increased to $10 \mathrm{mg}$ BID. There was a recurrence of ISB after two years which remitted when memantine was increased to $25 \mathrm{mg}$. Based on clinical follow ups and serial MOCAs over three years, it was determined that this patient did not have a major neurocognitve disorder.

\section{Conclusion}

ISB and its treatment is an understudied subject in individuals with intellectual disability. Resolution of new onset ISB in a 67 year old male with intellectual disability and comorbid mood disorder, is a previously unreported therapeutic effect of memantine. His ISB resolved with the addition of memantine $20 \mathrm{mg}$ daily after multiple failed trials of various classes of psychotropics. Two years later, reemergence of ISB subsided with memantine $25 \mathrm{mg}$ which is a higher than the recommended dose of this medication. Additional studies would be helpful in determining the maximum dose of this medication given its therapeutic efficacy at $25 \mathrm{mg}$ daily which was well tolerated with stable medical tests.

\section{Background}

Memantine is a non-competitive $\mathrm{N}$-methyl-d-aspartate receptor antagonist approved for the treatment of moderate to severe Alzheimer's disease (AD). However, knowledge of the involvement of glutamate pathways in multiple psychiatric disorders has expanded the therapeutic spectrum of memantine ${ }^{(1)}$. Monotherapy as well as combination with other medications has been found to be effective in a number of psychiatric conditions ${ }^{(1)}$.

Inappropriate sexual behavior (ISB) and its treatment is an understudied subject in individuals with intellectual disability (ID). Resolution of ISB in a 67 year old male with ID - a previously unreported therapeutic effect of memantine - is discussed here. At age 65, this patient manifested novel, ISB that was not controlled with various pharmacologic agents, and nonpharmacologic measures. While ISB was initially contained with high dose gabapentin, its dose had to be decreased due to poor tolerance. ISB 
finally resolved with the initiation of memantine, which was maintained at $10 \mathrm{mg}$ twice daily for two years. Thereafter, there was a recurrence of ISB which subsequently resolved at the increased dose of memantine $25 \mathrm{mg}$ daily, which was well tolerated. Based on clinical follow ups and serial MOCAs over three years, it was determined that this patient did not have a major neurocognitve disorder.

\section{Case Presentation}

A 67 year-old, single, white male with ID, hypertension, diabetes type 2 and hyperlipidemia followed up in psychiatry for many years for the treatment of intermittent explosive disorder and anxiety. He had been in special education classes through 12th grade. Per psychological disability evaluation (9.23.1998), he was determined to have a full scale IQ of 68 , indicative of mild mental retardation. His functional or adaptive capacities was determined to be below the established IQ level, and academic proficiency was uniformly at the first grade level. There was no significant family history of mental illness and no history of substance abuse. He lived by himself, went to a day program and had caregivers until 5:30pm. He was stabilized on low dose trifluoperazine and paroxetine, and later with paroxetine $50 \mathrm{mg}$ and gabapentin $300 \mathrm{mg}$ TID. Paroxetine was subsequently replaced with citalopram $40 \mathrm{mg}$ to help with symptoms.

Around age 65 , he developed new onset ISB towards men who were members of his program, and were mostly under 40 . His family stated that to their knowledge, the patient had not previously engaged in homosexual or heterosexual relationships. Incidents of ISB occurred in public bathrooms and other communal places. Per records, one of these episodes involved the intimate touching of penises with pants down (and no penetration), when interrupted by a supervisor. There was concern about the patient's escalating behavior because he was trying to pursue a relationship with this gentleman which was unwanted, but was not taking no for an answer. In other instances, he propositioned someone at a public pool, and placed himself very close to a man during an exercise class. He admitted to have done this because of urges. The patient's ISB became a source of complaint within the organization that had been supporting him. Subsequently, restrictions were enforced, basically excluding him until deemed safe to participate. A team was formulated with a behaviorist to assist in developing a safe program for him. The aim of the program was to reduce exposure to potential situations where sexual impulse may be a concern.

\section{Follow up and outcomes}

When risperidone $0.25 \mathrm{mg}$ BID was added, this behavior subsided temporarily but resumed. On $3 / 25 / 17$, risperidone was increased to $0.5 \mathrm{mg} \mathrm{BID}$, and on 3/28/17, gabapentin was gradually increased to $600 \mathrm{mg}$ TID, in combination with citalopram $40 \mathrm{mg}$. Subsequently, the undesirable behavior decreased and then subsided. However, the family was skeptical and keen on starting leuprolide injections, and patient was seen by this writer for a second opinion on $4 / 27 / 2017$. As he was stable, with no ISB for the past three weeks, leuprolide was not recommended. MOCA was 16/30 (with some preservation of executive function as in clock drawing, his short term recall was $3 / 5$ words). Labs included: Creatinine; 1.28, GFR $>60$, fasting glucose; 129, vitamin B12; 291, vitamin B1; 75, TPHA; negative. Vitamin B1 and B12 
supplements were started. Methylmalonate and other labs on 4/29/17 were normal. MRI brain (6/21/17) was unremarkable.

The patient was seen again on $6 / 27 / 17$ due to the family's concern about short term memory impairment (losing train of thought, could not figure out how to push the car seat back etc). His gait was unsteady. ISB had ceased about two months ago. It was noted that after gabapentin was increased to $600 \mathrm{mg}$ TID, he had become more confused, forgetful and unsteady. The patient was also taking risperidone $0.5 \mathrm{mg}$ BID and citalopram $40 \mathrm{mg}$ daily. MMSE was 17/30. Memantine was initiated at $5 \mathrm{mg}$ daily to be gradually increased to $10 \mathrm{mg}$ BID. A tapering regimen for gabapentin with $300 \mathrm{mg}$ BID and $600 \mathrm{mg}$ at pm was recommended. On 7/30/17, he became lethargic, had slurred speech and sustained falls. Subsequently, the dose of gabapentin was decreased to $600 \mathrm{mg}$ daily and later to $300 \mathrm{mg}$ daily. On $8 / 22 / 17$, the patient was noted to be doing well with improved cognition and resolution of ISB. He was taking memantine $30 \mathrm{mg}$ in two divided doses. As a higher than recommended dose was being administered mistakenly, memantine was decreased to $10 \mathrm{mg} \mathrm{BID.}$

After two years of stability, a recurrence of ISB was reported. The patient had begun pursuing a man in his program somewhat obsessively in a sexual manner over a two-month period. Per records, they had exposed themselves to each other in a bathroom during a movie. Although the patient acknowledged awareness of the consequences of his behavior, he continued to pursue this person and did what he could to ingratiate himself to him by offering food, and pushed the gentleman to act.

When he was examined on 4/9/19, there was no change in his MOCA score $(16 / 30)$. Recent creatinine was $1.27, \mathrm{GFR}>60$, and fasting glucose was 121 . Memantine was increased to $10 \mathrm{mg}$ in am and $15 \mathrm{mg}$ at pm to target ISB. At follow up visit on 5/21/19, no ISB had occurred after the dose of memantine was increased to $25 \mathrm{mg}$ daily. However, despite the control of ISB, there was a concern about belligerence and lying behavior. It was noted that the dose of risperidone had been mistakenly decreased to $0.25 \mathrm{mg} \mathrm{BID}$. At this visit, risperidone was increased back to $0.5 \mathrm{mg} \mathrm{BID}$, and given its high dose, citalopram $40 \mathrm{mg}$ was replaced with escitalopram $15 \mathrm{mg}$ daily. At follow up visit on 6/11/19, family expressed that patient's mood symptoms had resolved and he had continued remission of ISB. He had shown evidence of better judgment and had eventually agreed not to attend the LGBT parade, which he had intended to join in the hope of meeting somebody. He realized that this would be risky. Per collateral information on 1/28/2020, he was doing well with sustained resolution of ISB. His recent labs were normal with stable creatinine levels; GFR was $>60$.

On 3/7/20, patient presented as overall stable. In fact, MOCA had increased by two-points;(18/30). He had recently experienced urges of a sexual nature towards a female and had touched her inappropriately a few times during pool exercise classes. His case manager had closely questioned him, discussing the parameters that were outlined in the behavior program; red implied "serious uncontrollable urges", yellow " slight urges" and green "no urges ". After repeated denials, he admitted to experiencing slight urges. He subsequently took the initiative of suspending himself from the class as he realized that it was important to end the source of stimulation. His case manager's observation was that although he had become 
physically slow with age, there was no noticeable cognitive decline. Altered mental status had only occurred when high dose gabapentin was administered. Patient continued to work at the designated thrift stores and animal shelter. There were no concerns about behavior or difficulty in understanding instructions and task performance. Per last update in October 2020, patient continued to have stable mood and behavior, with no evidence of cognitive decline compared to his baseline.

\section{Discussion}

Older individuals with ID/mental retardation comprise a relatively unstudied group. In this population, the development of major neurocognitive disorder (NCD) is suspected in the context of new onset behavior disturbance, cognitive impairment, and a functional decline. As the emergence of NCD was suspected in this patient, due to novel onset ISB, memantine was initiated, and gabapentin was decreased due to cognitive and physical side effects. The latter resolved when its dose was gradually decreased from 1800 $\mathrm{mg}$ to $300 \mathrm{mg}$.

Per recent assessment in March, 2020, this writer questioned the patient's prior diagnosis of NCD due to lack of progression of cognitive decline. Additionally, he had demonstrated insight about his ISB and then successfully resolved it. Based on clinical follow ups and serial, stable MOCAs over three years, it was determined that this patient did not have a major neurocognitve disorder. It was postulated that new onset ISB was a manifestation of OCD in the setting of his chronic mood disorder associated with mental retardation. Patient was stabilized with high-dose Namenda at $25 \mathrm{mg}$ daily, in combination with other psychotropics.

Hypersexuality has been regarded as obsessive compulsive in nature, involving cortico-striatal circuits ${ }^{(2)}$, as in Huntington's disease ${ }^{(3)}$, Tourette's syndrome ${ }^{(4)}$, and Wilson's disease ${ }^{(5)}$. There is evidence of improvement of obsessive compulsive disorder (OCD) with memantine because the glutamatergic transmission of the cortico-striatal-thalamo-cortical circuitry is dysregulated in this condition. Based on this theory, a randomized, controlled study found significant improvement in OCD with the addition of memantine to fluvoxamine ${ }^{(6)}$, and also when memantine was combined with selective serotonin reuptake inhibitors and standalone therapy ${ }^{(1)}$. To corroborate this further, a key role of glutamate pathway abnormalities within the cortico-striatal-thalamo-cortical circuitry and temporal lobes was linked to the pathogenesis of $\mathrm{OCD}{ }^{(7,8)}$. It was noted that, by a de-excitation effect on the glutamatergic system in the temporal lobes and connected brain regions, memantine when combined with other psychotropics was clinically helpful in treating OCD symptoms ${ }^{(8,9)}$. Based on this evidence, it could be hypothesized that augmentation with memantine was helpful due to the above mechanism as this patient's ISB seemed to be somewhat obsessive compulsive.

Various neurobiological systems are involved in the etiology of ISB. These include the frontal lobes, the temporo-limbic network, the hypothalamus and the cortico-striatal circuits ${ }^{(2)}$. Aberrant function of the frontal lobes can precipitate disinhibited behavior and ISB ${ }^{(10)}$. The Kluver-Bucy syndrome, associated 
with autoerotic behavior and hyperorality is caused by bilateral lesions of the medial temporal lobe ${ }^{(11)}$. Hypersexuality has been associated with temporo-limbic strokes ${ }^{(12)}$, tumors ${ }^{(13)}$, and temporal lobe epilepsy ${ }^{(14)}$. Injury to the right hypothalamus and periventricular region can result in manic symptoms and increased libido ${ }^{(15)}$. ISB can be affected by androgens, estrogens, progesterone, prolactin, oxytocin, cortisol and pheromones. Neurotransmitters and neuropeptides such as nitric oxide, serotonin, dopamine, adrenaline, noradrenaline, opioids, acetylcholine, histamine and gamma-aminobutyric acid can also influence ISB ${ }^{(16)}$. Psychotropic agents including levodopa, benzodiazepines, and alcohol can induce agitation and sexual disinhibition ${ }^{(2)}$.

New onset ISB is generally associated with NCD. The prevalence of behavioral and psychiatric symptoms of minor and major neurocognitive disorders is $50-80 \%$, respectively ${ }^{(17)}$. A higher prevalence in residents of skilled nursing facilities and those with severe cognitive impairment has been documented $(18,19)$. In a study of 133 patients with NCD admitted to a geriatric psychiatry inpatient unit in Taiwan, 15 $\%$ manifested ISB before admission or during hospitalization ${ }^{(20)}$. Diagnosing NCD in adults with ID is difficult, due to the lack of standardized diagnostic criteria ${ }^{(21)}$. This patient was initially assessed to have NCD based on the DSM 5 criterion of impaired social cognition, as new onset ISB caused significant impairment in this domain. The ICD 10 criteria for NCD include a decline in emotional control, motivation, or a change in social behavior, manifested in at least one of the following: emotional lability, irritability, apathy or coarsening of social behavior ${ }^{(21)}$. A potential limitation in this case was the absence of formal neuropsychological testing for further evaluation. However, the patient's family was unwilling due to the COVID-19 pandemic. Additionally, there was no motivation to move forward with this due to the overall emotional and behavioral stability of the patient. However, as discussed previously, no evidence of NCD was noted at follow up and serial MOCAs over three years.

In the literature, the treatment of ISB has generally been described in the context of comorbid NCD. Antidepressants, anxiolytics, antipsychotics, anticonvulsants, cholinesterase inhibitors, hormonal agents/antiandrogens, and beta-blockers $(2,9,22)$ have been reported to be helpful for treating ISB in NCD. Gabapentin can cause reduced libido, anorgasmia, erectile dysfunction, and a reduction in $\operatorname{ISB}(2,23,24)$. This patient's ISB resolved when gabapentin was increased to $600 \mathrm{mg}$ TID in combination with risperidone and citalopram, but it was poorly tolerated and needed to be decreased to $300 \mathrm{mg}$ daily. Escitalopram $15 \mathrm{mg}$ later replaced citalopram $40 \mathrm{mg}$ due to safety concerns, and its efficacy seemed comparable to citalopram in treating his chronic psychiatric symptoms. Risperidone, which was also a long term medication (taken off and on since 2014), stabilized his primary mood symptoms at the slightly increased dose of $0.5 \mathrm{mg}$ BID. It is pertinent to document that the recurrence of ISB resolved soon after increasing the dose of memantine to $25 \mathrm{mg}$ which occurred long before the above changes were implemented.

Recently, knowledge of the involvement of glutamate pathways in multiple psychiatric disorders broadened the therapeutic spectrum of memantine. In a double-blind clinical trial study of 40 patients with attention deficit hyperactivity disorder (ADHD), aged 18 to 45 years, memantine was therapeutic in 
decreasing symptoms of inattention, memory impairment, hyperactivity, impulsivity and emotional lability (25). It was also effective in improving symptoms of autism spectrum disorder ${ }^{(26)}$. This aspect merited discussion as this patient did not have co morbid ADHD or autism. Other conditions such as OCD, bipolar mania, binge eating disorder, PTSD, generalized anxiety disorder, negative symptoms of schizophrenia and catatonia also benefited with memantine ${ }^{(1)}$.

This patient's ISB diminshed when memantine was initiated, and resolved at $20 \mathrm{mg}$ daily. Two years later, there was a recurrence of ISB which ceased when memantine was increased to $10 \mathrm{mg}$ in am and $15 \mathrm{mg}$ at pm. He tolerated the slightly higher dose of memantine with stable creatinine and GFR. In reviewing its formulations, memantine ER $28 \mathrm{mg}$ allows for a higher total daily dose compared to the recommended maximum dose of memantine IR $10 \mathrm{mg}$ BID. Moreover, pharmacokinetic data indicates greater exposure with the ER formulation, but thus far its clinical implication is unclear, as these formulations have not been assessed in a comparative clinical trial ${ }^{(27)}$.

Memantine is excreted primarily in urine as an unchanged parent drug (about $50 \%$ ), in part via renal tubular secretion, and has a half-life of 60 to 80 hours. The remainder of memantine that is not excreted as unchanged is converted to the polar metabolites(memantine glucuronide conjugate (about 25\%), 6hydroxy-memantine, and 1-nitroso-deaminated memantine, which manifest minimal NMDA receptor antagonistic activity). In vitro studies indicate that memantine produces no or minimal inhibition of the cytochrome P450 isozymes.

Based on the predicted steady-state plasma concentrations with current dosing regimen of $10 \mathrm{mg}$ twice daily, no dosage adjustments are required for patients with mild or moderate renal impairment. A target dose of $5 \mathrm{mg}$ twice daily is recommended in those with severe renal impairment ${ }^{(28,29)}$. The data comparing $10 \mathrm{mg} \mathrm{BID}$ and 20mg QD of the IR formulation, support use of once daily IR dosing; IR is prescribed as QD in Europe ${ }^{(30)}$.

It is interesting to note that this patient tolerated a dose of memantine $30 \mathrm{mg}$ daily that was mistakenly administered for about 27 days, in 2017, when initially prescribed. It was immediately decreased to $20 \mathrm{mg}$ daily when this error was identified.

Non pharmacological management of ISB should be considered as first line due to ethical and safety concerns. However, pharmacological strategies are often combined as generally non pharmacologic treatment alone has been found to be ineffective. These measures include environmental measures (e.g. decreased media and other types of stimulation), behavioral (redirection, activities, crafts, stuffed animals), educational and cognitive-behavioral therapy $(22,31)$. Families and nursing staff should be closely involved in such treatment plans. ISB is a common, but understudied issue for individuals with developmental disabilities, intellectual disability, and/or acquired brain injuries. The results of a systemic review indicated that behavior analytic approaches were effective for ISB, and that function-based interventions had strong supporting evidence in this population ${ }^{(32)}$. 
Non pharmacological treatment was ineffective for this patient initially, but he was more open to education about social norms and redirection when stabilized with memantine. The aim of the patient's behavior program was to reduce exposure to potential situations where sexual impulse may be a concern.

\section{Conclusion}

Memantine could be a promising medication for the control of ISB in patients with ID when used as an adjunct. ISB and its treatment is an understudied subject in such individuals, and given that memantine was added to this patient's existing psychotropics, further studies could determine whether monotherapy with memantine could resolve ISB in these patients. Additional studies would be helpful in determining the maximum dose of this medication given its therapeutic efficacy at $25 \mathrm{mg}$ daily which was well tolerated with stable medical tests. Resolution of new onset inappropriate sexual behavior (ISB) in a 67 year old male with intellectual disability and comorbid mood disorder, is a previously unreported therapeutic effect of memantine. His ISB which was not associated with NCD, resolved with the addition of memantine $20 \mathrm{mg}$ daily after multiple failed trials of various classes of psychotropics. Two years later, reemergence of ISB subsided with memantine $25 \mathrm{mg}$ which is a higher than the recommended dose of this medication.

\section{Abbreviations}

ISB: Inappropriate sexual behavior

ID: Intellectual disability

AD: Alzheimer's Disease

IQ: Intelligence Quotient

NCD: Major Neurocognitive Disorder

GFR: Glomerular Filtration Rate

BID: Twice Daily

QD: Once Daily

IR: Immediate Release

ER: Extended Release

OCD: Obsessive Compulsive Disorder

MOCA: Montreal Cognitive Assessment 
MMSE: Mini-Mental State Exam

MRI: Magnetic Resonance Imaging

TPHA: Treponema Pallidum Hemagglutination Assay

LGPT: Lesbian Gay Bisexual Transgender

\section{Declarations}

\section{Ethics approval and consent to participate:}

NA

\section{Consent for publication:}

Written informed consent for publication of their clinical details and/or clinical images was obtained from the patient/parent/guardian/ relative of the patient. A copy of the consent form is available for review by the Editor of this journal.

\section{Availability of data and material:}

NA. This is a case report and patient provided consent

\section{Competing interests:}

No competing interests

\section{Funding:}

NA

\section{Authors' contributions:}

I am the sole author, and give final approval for this version to be published

\section{Acknowledgements:}

None

\section{References}

1. Lu S, Nasrallah HA. The use of memantine in neuropsychiatric disorders: An overview. Ann Clin Psychiatry. 2018 Aug;30 (3):234-248.

2. De Giorgi R, Series H. Treatment of Inappropriate Sexual Behavior in Dementia. Curr Treat Options Neurol. 2016; 18: 41. 
3. Janati A. Kluver-Bucy syndrome in Huntington's chorea. J Nerv Ment Dis. 1985;173(10):632-5.

4. Comings DE, Comings BG. A case of familial exhibitionism in Tourette's syndrome successfully treated with haloperidol. Am J Psychol. 1982;139(7):913-5.

5. Akil M, Brewer GJ. Psychiatric and behavioral abnormalities in Wilson's disease. Adv Neurol. 1995;65:171-8.

6. Ghaleiha A, Entezari N, Modabbernia A, Najand B, Askari $\mathrm{N}$ et al. Memantine add-on in moderate to severe obsessive-compulsive disorder: randomized double-blind placebo-controlled study. J Psychiatr Res. 2013 Feb;47 (2):175-80.

7. Fettes P, Schulze L, Downar J. Cortico-Striatal-Thalamic Loop Circuits of the Orbitofrontal Cortex: Promising Therapeutic Targets in Psychiatric Illness. Front Sys Neurosci. 2017.27;11:25.

8. Vlček PPolák J, Brunovský M, Horáček J. Role of Glutamatergic System in Obsessive-Compulsive Disorder with Possible Therapeutic Implications. Pharmacopsychiatry. 2018 Nov; 51(6):229-242.

9. Ibrahim C, Reynaert C. Hypersexuality in neurocognitive disorders in elderly people-a comprehensive review of the literature and case study. Psychiatr Danub. 2014;26 (Suppl 1):36-40.

10. Bardell A, Lau T, Fedoroff JP. Inappropriate sexual behavior in a geriatric population. Int Psychogeriatr. 2011;23 (7):1182-8.

11. Lilly R, Cummings JL, Benson DF, Frankel M. The human Klüver-Bucy Ibrahim C, Reynaert C. Hypersexuality in neurocognitive disorders in elderly people-a comprehensive review of the literature and case study. Psychiatr Danub. 2014;26 (Suppl 1):36-40.

12. Monga TN, Monga M, Raina MS, Hardjasudarma M. Hypersexuality in stroke. Arch Phys Med Rehabil. 1986;67(6):415-7.

13. Blustein J, Seeman M V. Brain tumors presenting as functional psychiatric disturbances. Can Psychiatr Ass J. 1972;17(2), Suppl 2:SS59.

14. Bear DM, Fedio P. Quantitative analysis of interictal behavior in temporal lobe epilepsy. Arch Neurol. 1977;34 (8):454-67.

15. Miller BL, Cummings JL, Mclntyre H, Ebers G, Grode M. Hypersexuality or altered sexual preference following brain injury. J Neurol Neurosurg Psychiatry. 1986;49 (8):867-73.

16. Meston CM, Frohlich PF. The neurobiology of sexual function. Arch Gen Psychiatry. 2000;57(11):1012-30.

17. Lyketsos CG, Lopez O, Jones B, Fitzpatrick AL, Breitner J, DeKosky S.Prevalence of neuropsychiatric symptoms in dementia and mild cognitive impairment: results from the cardiovascular health study. JAMA. 2002 Sep 25;288(12):1475-83.

18. Szasz G. Sexual incidents in an extended care unit for aged men. J Am Geriatr Soc. 1983 Jul;31(7):407-11.

19. Burns A, Jacoby R, Levy R. Psychiatric phenomena in Alzheimer's disease. IV: Disorders of behaviour. Brit J Psychol. 1990;157:86-94. 
20. Tsai SJ, Hwang JP, Yang CH, Liu KM, Lirng JF. Inappropriate sexual behaviors in dementia: a preliminary report. Alzheimer Dis Assoc Disord. 1999 Jan;13(1):60-2.

21. Aylward EH, Burt DB, Thorpe LU, Lai F, Dalton A. Diagnosis of dementia in individuals with intellectual disability. Joumal of Intellectual Disability Research. Vol 41, Part 2,152-164. 1997.

22. Joller P, Gupta N, Seitz DP, Frank C, Gibson M, Gill SS. Approach to inappropriate sexual behaviour in people with dementia. Can Fam Physician. 2013;59 (3):255-60.

23. Alkhalil C. Hahar N, Alkhalil B , Zavros G, Lowenthal DT. Can gabapentin be a safe alternative to hormonal therapy in the treatment of inappropriate sexual behavior in demented patients? Int Urol Nephrol. 2003; 35(2):29-302.

24. Alkhalil C, Tanvir F, Alkhalil B, Lowenthal DT. Treatment of sexual disinhibition in dementia: case reports and review of the literature. Am J Ther. 2004;11(3):231-5.

25. Mohammadzadeh S, Ahangari TK, Yousefi F. The effect of memantine in adult patients with attention deficit hyperactivity disorder. Hum Psychopharmacol 2019 Jan;34(1):2687.

26. Karahmadi M, Tarrahi MJVatankhah Ardestani SS, Omranifard V, Farzaneh B. Efficacy of Memantine as Adjunct Therapy for Autism Spectrum Disorder in Children Aged <14 Years. Adv Biomed Res. 2018 Oct $1 ; 7: 131$.

27. Plosker GL. Memantine extended release ( $28 \mathrm{mg}$ once daily): a review of its use in Alzheimer's disease. Drugs. 2015 May;75 (8):887-97.

28. Periclou A, Ventura D, Rao N, Abramowitz W. Pharmacokinetic study of memantine in healthy and renally impaired subjects. Clin Pharmacol Ther. 2006 Jan;79(1):134-43.

29. Moritoyo T, Hasunuma T, Harada K, Tateishi T, Watanabe M, Kotegawa T, et al.

Effect of renal impairment on the pharmacokinetics of memantine. Pharmacol Sci. 2012;119(4):3249.

30. Puzantian T, Carlat DJ, in Medication Fact Book for Psychiatric Practice, fifth ed, Carlat publishing, 2020. LLC.Newburyport, MA. p 103

31. Tune LE, Rosenberg J. Nonpharmacological treatment of inappropriate sexual behavior in dementia: the case of the pink panther. Am J Geriatr Psychiatry. 2008;16 (7):612-3.

32. Clay CJ, Bloom SE, Lambert JM. Behavioral Interventions for ISB in Individuals with developmental disabilities, intellectual disability and/or acquired brain injuries ( $\mathrm{ABI}$, and Acquired Brain Injury: $\mathrm{A}$ Review. American Journal on Intellectual and developmental disabilities, 2018, vol. 123, No. 3, 254282

\section{Supplementary Files}

This is a list of supplementary files associated with this preprint. Click to download.

- CAREchecklistEnglish20133.docx 